\title{
Data approximation using Lotka-Volterra models and a software minimization function
}

\author{
M. FEČKAN AND J. PAČUTA
}

\begin{abstract}
In recent years, a lot of effort has been put into finding suitable mathematical models that fit historical data set. Such models often include coefficients and the accuracy of data approximation depends on them. So the goal is to choose the unknown coefficients to achieve the best possible approximation of data by the corresponding solution of the model. One of the standard methods for coefficient estimation is the least square method. This can provide us data approximation but it can also serve as a starting method for further minimizations such as Matlab function fminsearch.
\end{abstract}

Mathematics Subject Classification 2010: 34C60, 65C20, 91B64.

General Terms: Algorithms, Experimentation

Keywords: Systems modelling, linear regression, error minimization, integral method

\section{INTRODUCTION}

The general goal of modelling is to find a simple model that fits a historical data set of some property and indicates how could this property evolve in future. In this paper using given data set, we estimate parameter values in a classic Lotka-Volterra model. These parameter values describe various interactions between populations, for example, growth, competition, mutualism or predation. The model is fully determined by its parameters so that they need to be determined to apply the model in practice. Therefore the problem is to compute the parameter values of a model as accurately as possible in order to fit the raw data as closely as possible.

To achieve this goal, various methods have been discussed, for example Shatalov et al. in [1], and Fedatov and Shatalov in [2] started an approach of using direct integration of equations in Lotka-Volterra system and apply quadrature rules to obtain the unknown parameters. Michalakelis et al. in [3] use artificial intelligence methods to solve a non-linear system. Our work is connected with results of Kloppers and Greef in [4] who compare the integral method, the log integral method and advanced

M.F. was supported by the Slovak Research and Development Agency under the contract No. APVV-180308 and by the Slovak Grant Agency VEGA No. 2/0153/16.

J.P. was supported by the Slovak Research and Development Agency under the contract No. APVV-18-0308 and by the Slovak Grant Agency VEGA No. 1/0347/18. 
method from [3].

In this work, we use Matlab to approximate historical data by solutions of a LotkaVolterra model

$$
\frac{d x_{i}}{d t}=x_{i}\left(p_{i 1}+\sum_{j=1}^{m} p_{i, j+1} x_{j}\right), \quad i=1, \ldots, m .
$$

We use two historical data sets to estimate parameter values in the system (1). First, we compute coefficients $p_{i j}, i=1, \ldots, m, j=1, \ldots, m+1$ in the system (1) by using numerical integration along with least squares method. Unlikely in [4], we use these $p_{i j}$ as an initial point for fminsearch - Matlab function for finding minima of functions. We use this Matlab function to find the coefficients $P_{i j}$ such that the corresponding numerical solution of (1) is discreetly closest to historical data in terms of least squares. Similar approach - minimization of some error functional was previously used in e.g. [3] where a genetic algorithm was applied. Finally, we compare this optimal numerical solution of (1) with $P_{i j}$ with the solution of (1) with initial coefficients $p_{i j}$ (i.e. without using fminsearch).

\section{DESCRIPTION OF METHODS}

Assume $m=3$ in the Lotka Volterra model (1). The system is then represented by

$$
\begin{aligned}
& \frac{d x}{d t}=x\left(p_{11}+p_{12} x+p_{13} y+p_{14} z\right), \\
& \frac{d y}{d t}=y\left(p_{21}+p_{22} x+p_{23} y+p_{24} z\right), \\
& \frac{d y}{d t}=z\left(p_{31}+p_{32} x+p_{33} y+p_{34} z\right) .
\end{aligned}
$$

The unknown functions $x, y, z$ are three species that compete for available resources in a system.

Denote $D=D_{i j}, i=1, \ldots, n, j=1,2,3$ the historical data set (Figure 1). In our case, we consider prices of three crypto currencies during $n$-day period.

Now, we will in short explain the integral method for obtaining unknown initial coefficients $p_{i j}$ using historical data $D$; see [4] for details. We choose the first differential equation in (2) and integrate both sides with respect to $t$ over the interval $[k, k+1]$ for $k=1,2, \ldots, n-1$. We approximate every integral by applying the Trapezium rule. For example, the integral $\int_{k}^{k+1} x(t) \mathrm{d} t$ is approximated by $(x(k+1)-x(k)) / 2$ what is equal to $\left(D_{k 1}+D_{k+1,1}\right) / 2$. The system (2) then turns into 


\begin{tabular}{|r|r|r|r|}
\hline \multicolumn{1}{|c|}{ date } & \multicolumn{1}{c|}{ eth } & \multicolumn{1}{c|}{ dash } & \multicolumn{1}{c|}{ xmr } \\
\hline 15-Mar-18 & 614.84 & 425.95 & 215.84 \\
\hline 16-Mar-18 & 611.78 & 418.54 & 213.21 \\
\hline 17-Mar-18 & 601.68 & 426.3 & 215.49 \\
\hline 18-Mar-18 & 551.64 & 388.18 & 197.43 \\
\hline 19-Mar-18 & 546.63 & 392.32 & 210.33 \\
\hline 20-Mar-18 & 556.72 & 412.49 & 217.94 \\
\hline 21-Mar-18 & 559.1 & 429.15 & 224.26 \\
\hline 22-Mar-18 & 562.1 & 434.44 & 218.56 \\
\hline 23-Mar-18 & 539.86 & 411.27 & 212.47 \\
\hline 24-Mar-18 & 542.57 & 432.17 & 215.37 \\
\hline 25-Mar-18 & 522.7 & 420.02 & 207.32 \\
\hline 26-Mar-18 & 524.29 & 410.73 & 211.79 \\
\hline 27-Mar-18 & 489.59 & 390.24 & 196.85 \\
\hline 28-Mar-18 & 450.29 & 354.93 & 187.47 \\
\hline 29-Mar-18 & 448.08 & 350.85 & 199.31 \\
\hline 30-Mar-18 & 385.9 & 328.65 & 176 \\
\hline 31-Mar-18 & 395 & 310.91 & 172.53 \\
\hline 01-Apr-18 & 397.25 & 306.21 & 178.78 \\
\hline 02-Apr-18 & 379.7 & 292.64 & 175.87 \\
\hline 03-Apr-18 & 387.31 & 312.64 & 177.57 \\
\hline
\end{tabular}

Fig. 1. Price data in dollars

a system of linear equations

$$
\left[\begin{array}{c}
b_{1} \\
b_{2} \\
\vdots \\
b_{n-1}
\end{array}\right]=\left[\begin{array}{cccc}
a_{11} & a_{12} & a_{13} & a_{14} \\
a_{21} & a_{22} & a_{23} & a_{24} \\
\vdots & & & \\
a_{n-1,1} & a_{n-1,2} & a_{n-1,2} & a_{n-1,2}
\end{array}\right] \cdot\left[\begin{array}{c}
p_{11} \\
p_{12} \\
p_{13} \\
p_{14}
\end{array}\right]
$$

or $B=A . p_{1}$ where $B=b_{k}=D_{k+1,1}-D_{k 1}, p_{1}=p_{1 j}(j=1,2,3,4), A=a_{k j}$ and $a_{k 1}=\left(D_{k 1}+D_{k+1,1}\right) / 2, a_{k j}=\left(D_{k 1} D_{k j}+D_{k+1,1} D_{k+1, j}\right) / 2, j=2,3,4$. The matrix $p_{1}$ contains the unknown coefficients. If, in addition, the matrix $A^{\top} A$ is regular $\left(A^{\top}\right.$ denotes the transpose of $A$ ) then $p_{1}$ is given by

$$
p_{1}=\left(A^{\top} A\right)^{-1} A^{\top} B .
$$

\section{EXAMPLES}

\subsection{Example 1}

In this example, we use historical price data of crypto currencies Ethereum, Dash and Monero that are competitors $x, y, z$ respectively. The data are downloaded from https://coinmetrics.io and the date range of the data is from 15th March 2018 till 3rd April 2018; see Figure 1. As can be seen in Figure 2, our data are volatile and not very 

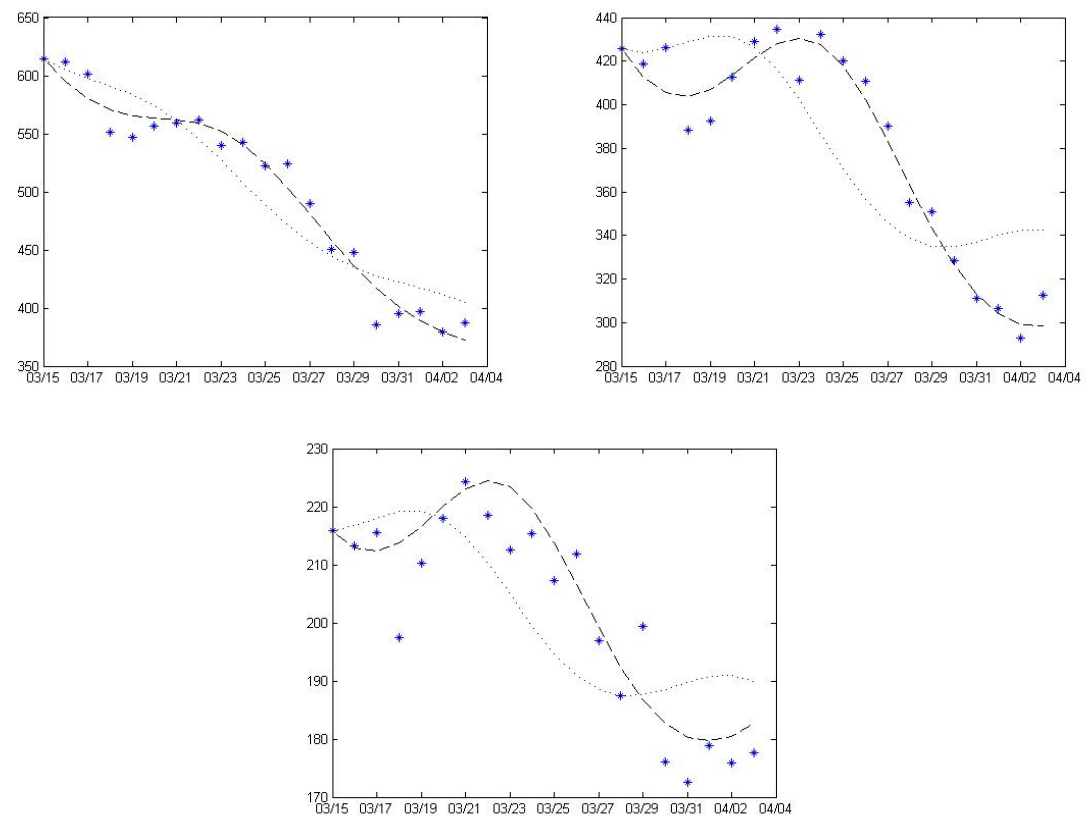

Fig. 2. From left to right: approximation of Ethereum, Dash and Monero currency prices. The asterisks represent real data, dashed lines are solutions of (1) optimized by fminsearch and dotted lines are solutions of (1) computed only by least square method.

suitable for approximation by solutions of model (1). Our choice is due to the problem that suitable data are usually not freely available. Despite of this, it is also seen that the approximation of such bad data using Matlab function fminsearch is better than the approximation by least square method without using fminsearch.

Applying least square method along with fminsearch, we obtained coefficients $P_{i j}$ and the resulting system is given by

$$
\begin{aligned}
& \frac{d x}{d t}=x(-0.3844+0.0000 x-0.0014 y+0.0044 z), \\
& \frac{d y}{d t}=y(-0.6470-0.0001 x-0.0025 y+0.0079 z), \\
& \frac{d y}{d t}=z(-0.3419+0.0002 x-0.0020 y+0.0049 z) .
\end{aligned}
$$

To determine mutual interactions between competitors, we can look at coefficients of mixed terms $x y, y z$ and $x z$; see [5]. The equal signs of coefficients $P_{41}, P_{23}$ indicate that $x$ is cooperating with $z$ and when the population of $x$ decreases rapidly then also 


\begin{tabular}{|r|r|r|r|}
\hline \multicolumn{1}{|c|}{ Period } & \multicolumn{1}{c|}{$\mathbf{x}$} & \multicolumn{1}{l|}{$\mathbf{y}$} & \multicolumn{1}{l|}{$\mathbf{z}$} \\
\hline Jan-98 & 0.52 & 0.15 & 0.33 \\
\hline Jul-98 & 0.47 & 0.21 & 0.32 \\
\hline Jan-99 & 0.43 & 0.27 & 0.3 \\
\hline Jul-99 & 0.4 & 0.31 & 0.29 \\
\hline Jan-00 & 0.38 & 0.35 & 0.27 \\
\hline Jul-00 & 0.37 & 0.36 & 0.27 \\
\hline Jan-01 & 0.36 & 0.37 & 0.27 \\
\hline Jul-01 & 0.36 & 0.37 & 0.27 \\
\hline Jan-02 & 0.35 & 0.38 & 0.27 \\
\hline Jul-02 & 0.36 & 0.38 & 0.26 \\
\hline Jan-03 & 0.37 & 0.39 & 0.24 \\
\hline Jul-03 & 0.38 & 0.39 & 0.23 \\
\hline Jan-04 & 0.39 & 0.39 & 0.22 \\
\hline Jul-04 & 0.38 & 0.39 & 0.23 \\
\hline Jan-05 & 0.37 & 0.39 & 0.24 \\
\hline Jul-05 & 0.38 & 0.39 & 0.23 \\
\hline Jan-06 & 0.38 & 0.4 & 0.22 \\
\hline Jul-06 & 0.36 & 0.39 & 0.25 \\
\hline Jan-07 & 0.34 & 0.38 & 0.28 \\
\hline
\end{tabular}

Fig. 3. Share data

$z$ decreases. Signs of coefficients $P_{42}$ and $P_{33}$ indicate that $y$ is predating on $z$. The population of $y$ strongly reflects the population of $z$. Of course in reality, the system of interactions between competitors is not isolated, since the prices of our three crypto currencies coincide with prices of hundreds of others, with economical and political changes etc.

\subsection{Example 2}

In this example, we use historical data originally used in [3] and also used in [4]. The data represent shares of three telephone service providers (competitors) (see Figure 3) and the sum of these shares in each row is equal to 1 . We can look at any of these competitors from two different points of view:

(1) The competitor competes with each of two species and both them compete to each other, i.e. three mutual competitors in one system - the same approach as in Example 1.

(2) The competitor competes with the rest of the species (the two other species form one competitor), i.e. only two competitors in one system - we sum the values of the shares of same date in columns for competitors $y, z$ and then find the coefficients of model (1) with $m=2$ using fminsearch.

In Figure 4, we see that the second approach still provides us a good approximation of historical data values, especially in case of competitors $x$ and $y$. 

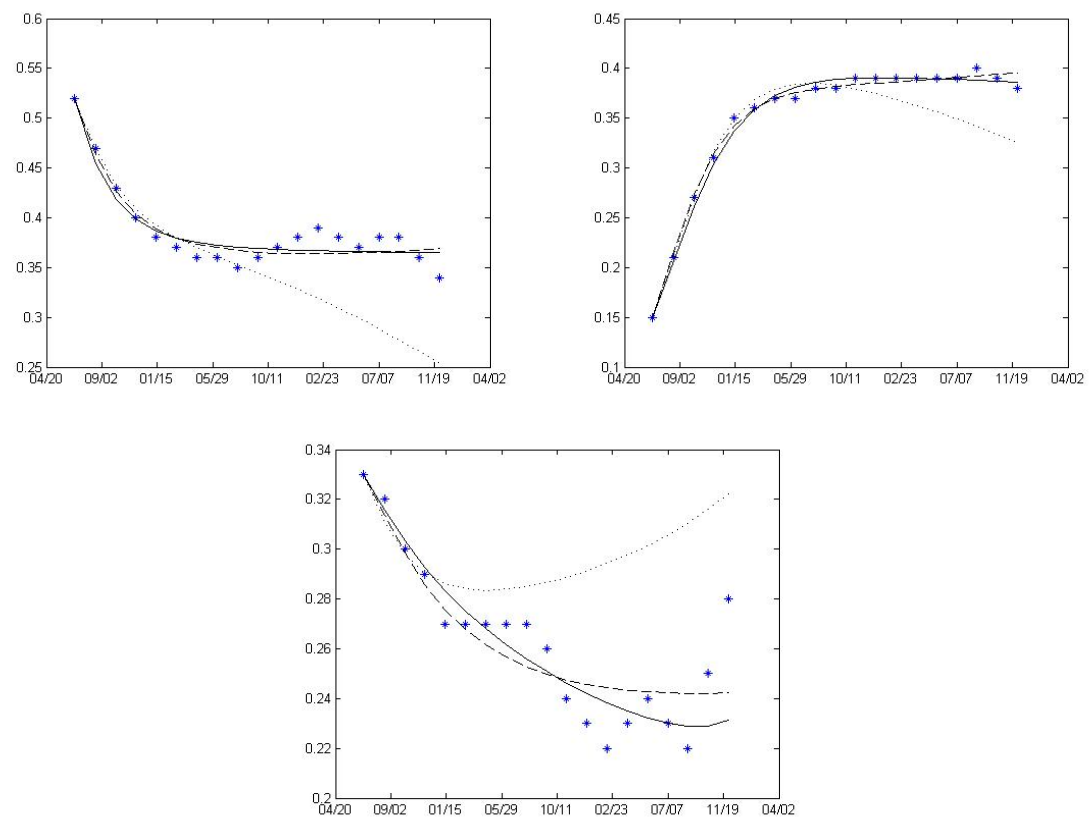

Fig. 4. From left to right: approximation of telephone service providers $x, y, z$. The asterisks represent real data, dashed lines are solutions of (1) with $m=3$ (i.e. first point of view), full lines are solutions of (1) with $m=2$ (i.e. second point of view) and dotted lines are initial solutions of (1) without using fminsearch.

The case $z$, however, is not so clear. Comparing the values of error function (i.e. sum of squares of differences of data and solutions of system 1 with optimized coefficients $P_{i j}$ ) we come to conclusion: second approach did slightly better than first one: In the first approach, the minimum of the error function is 0.0079 and fminsearch found it in 10.806295 seconds, in the second approach, the minimum of the error function is 0.0056 and fminsearch found it in 4.166628 seconds.

\section{CONCLUSIONS}

(1) As illustrated in Example 1, Matlab function fminsearch provides us better approximations than only the least square method. fminsearch is a robust however relatively slow function and one can consider using a different function for finding minima especially for large data set.

(2) For volatile data set such as prices, it may be better to use this method along stochastic calculus to achieve better approximations. 
(3) Example 2 shows us that competition of one species with the rest of species (the second approach in Example 2) may lead to similar results as mutual competition of three species (the first approach). The second one can be even better for small data set, since only minimization of a function of six variables is required instead of twelve. Obviously, this costs less counting time (more than twice less in our case).

\section{APPENDIX}

In the Appendix, we provide the Matlab source code we use in our paper. Function "est" is the main function that calculates initial coefficients $p_{i j}$ by using least squares method (function "Param") then calculates optimized $P_{i j}$ with fminsearch and compares corresponding solutions of Lotka - Volterra models with both $p_{i j}$ and $P_{i j}$.

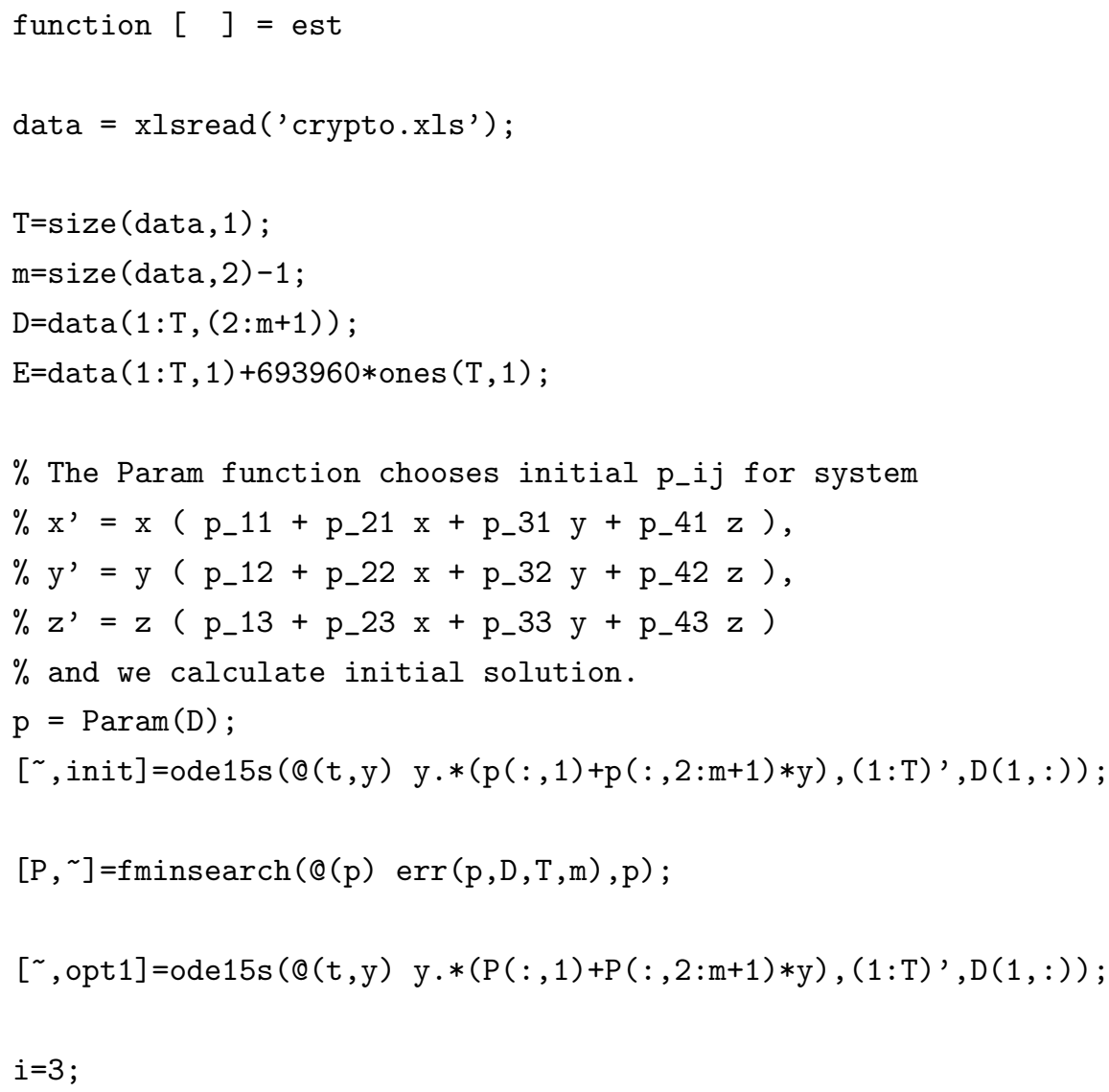




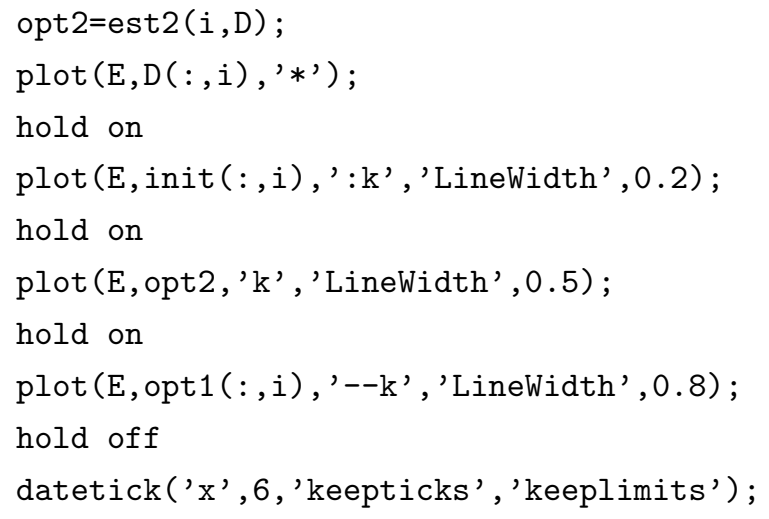

Function "Param" uses least squares method to obtain initial values $p_{i j}$ and contains fix for the case of badly conditioned matrix $A^{\top} A$.

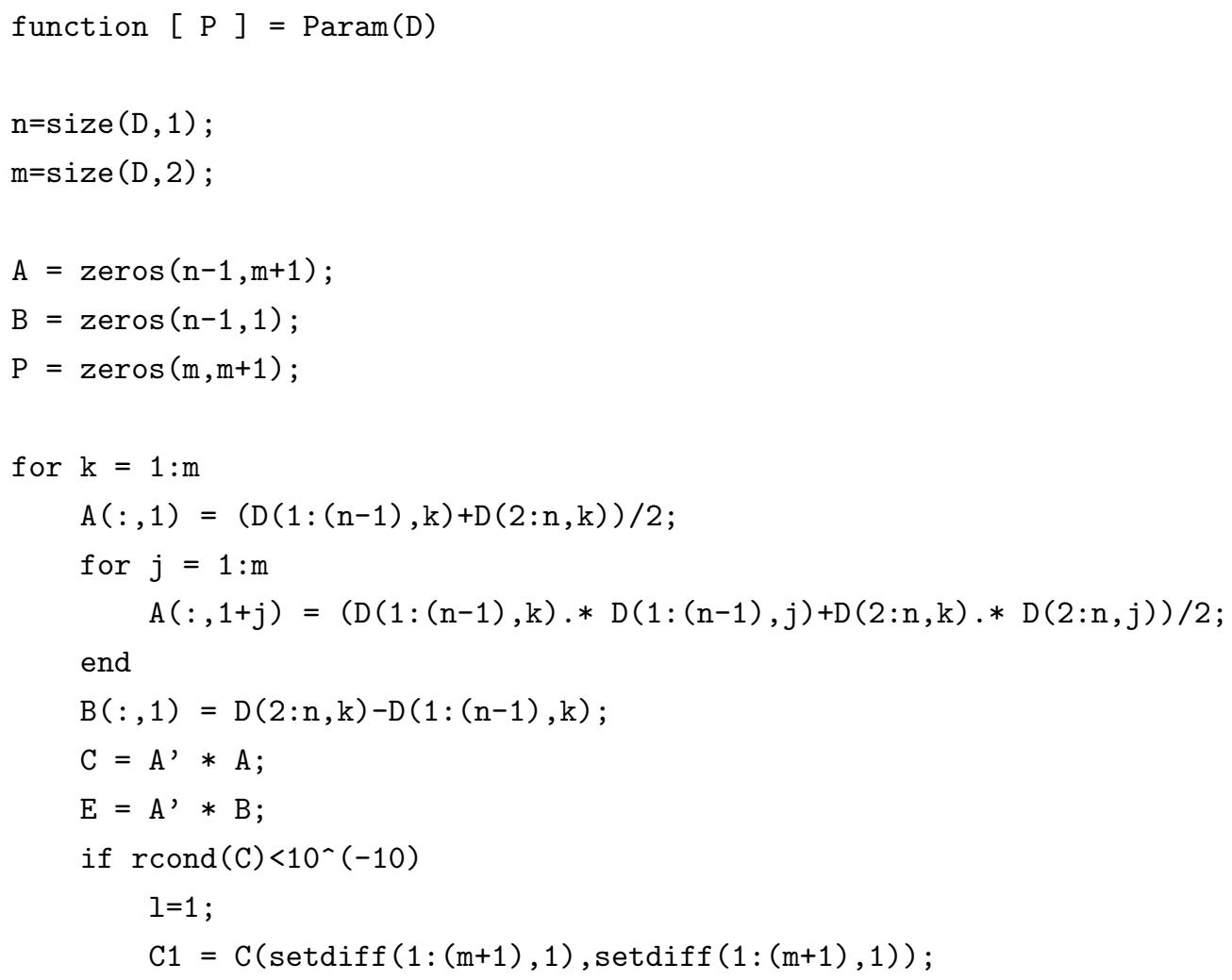




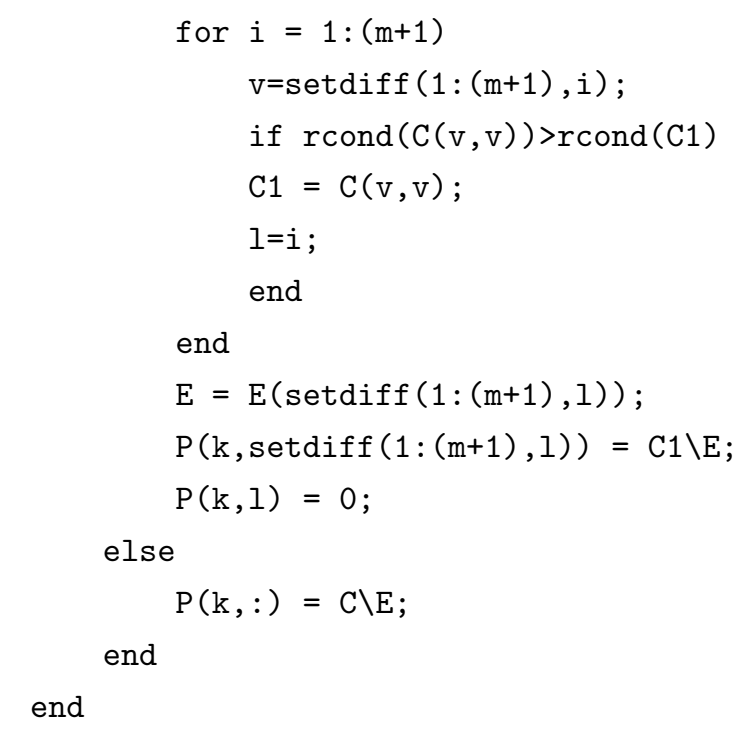

Function "est2" is similar to "est", however is based on the second approach from Example 2.

function $[$ opt $]=\operatorname{est2}(i$, data)

$\mathrm{T}=\operatorname{size}(\operatorname{data}, 1)$;

$\mathrm{m}=\operatorname{size}($ data, 2$)$;

$\mathrm{D}=\operatorname{zeros}(\mathrm{T}, 2)$;

$D(:, 1)=\operatorname{sum}(\operatorname{data}(1: T, \operatorname{set} \operatorname{diff}(1: m, i)), 2)$;

$D(:, 2)=\operatorname{sum}(\operatorname{data}(1: T, i), 2)$;

$\mathrm{p}=\operatorname{Param}(\mathrm{D})$;

$[P, \sim]=f \operatorname{minsearch}(@(p) \operatorname{err}(\mathrm{p}, \mathrm{D}, \mathrm{T}, 2), \mathrm{p})$;

$[\sim$, opt 0$]=\operatorname{ode1} 15 \mathrm{~s}(@(t, y)$ y.*(P(:,1)+P(:,2:3)*y), (1:T), $\mathrm{D}(1,:))$;

opt $=$ opt $0(:, 2)$;

end

Function "err" represents the error functional that is minimized by fminsearch.

function [ value ] $=\operatorname{err}(\mathrm{p}, \operatorname{data}, \mathrm{T}, \mathrm{m})$

$[\sim, y]=\operatorname{ode15s}\left(@(t, y) y \cdot *(p(:, 1)+p(:, 2: m+1) * y),(1: T)^{\prime}, \operatorname{data}(1,:)\right)$; $\mathrm{n}=\operatorname{size}(\mathrm{y}, 1)$; 
value $=\operatorname{sum}\left(\operatorname{sum}\left((y-\operatorname{data}(1: n,:)) \cdot{ }^{\wedge} 2\right)\right) ;$

end

\section{REFERENCES}

M. Shatalov, J.C. Greeff, I. Fedotov, S.V. Joubert: Parametric identification of the model with one predator and two prey species. TIME1008 International Conference Proceedings, (2008), 101-110.

M. Shatalov, I. Fedotov: On identification of dynamical system parameters from experimental data. Research Group in Mathematical Inequalities and Applications 10(1) (2007) 1-9.

C. Michalakelis, T.S. Sphicopoulos, D. Varoutas: Modelling competition in the telecommunications market based on the concepts of population biology. Transactions on Systems, Man and Cybernetics, Part C: Applications and Reviews 4 (2011) 200-210.

P.H. Kloppers, J.C. Greeff: Lotka-Volterra model parameter estimation using experimental data. Applied Mathematics and Computation 224 (2013) 817-825.

Y. Takeuchi: Global Dynamical Properties of Lotka-Volterra Systems. Singapore: World Scientific Publishing Company, 1996.

\section{Michal Fečkan}

Department of Mathematical Analysis and Numerical Mathematics, Faculty of Mathematics, Physics and Informatics, Comenius University in Bratislava, Mlynská dolina, 84248 Bratislava, Slovakia Mathematical Institute of Slovak Academy of Sciences, Štefánikova 49, 81473 Bratislava, Slovakia e-mail: Michal.Feckan@fmph.uniba.sk

\section{Július Pačuta}

Department of Mathematical Analysis and Numerical Mathematics, Faculty of Mathematics, Physics and Informatics, Comenius University in Bratislava, Mlynská dolina, 84248 Bratislava, Slovakia e-mail: julius.pacuta@fmph.uniba.sk 